\title{
Modeling the Prospects of Plug-In Electric Buses to Reduce GHG Emissions and Cost While Meeting Route Demands: A Case Study of the "Unitrans" Bus Fleet Serving the Davis, California Urbanized Area
}

\author{
Kurt Kornbluth*, Colin Mickle, Kelley Hestmark \\ University of California Davis, Davis, USA \\ Email: "kkorn@ucdavis.edu, hestmakv@gmail.com, colin.mickle@gmail.com \\ Received 18 April 2016; accepted 16 May 2016; published 19 May 2016 \\ Copyright (C) 2016 by authors and Scientific Research Publishing Inc. \\ This work is licensed under the Creative Commons Attribution International License (CC BY). \\ http://creativecommons.org/licenses/by/4.0/ \\ (c) (i) Open Access
}

\begin{abstract}
As university campuses look to decrease their greenhouse gas emissions, plug-in electric buses may provide a low carbon alternative to conventionally fossil-powered buses. This study investigates the viability for Unitrans, the bus service for the greater Davis area and the university campus, to replace current compressed natural gas buses with plug-in electric versions. This study presents an inventory of market available electric buses, their associated costs, incentives, and infrastructure concerns, and compares projected energy use, net present cost, and greenhouse gas emissions with their CNG counterparts. ADVISOR vehicle simulation software is used to estimate the energy use of a typical electric bus (New Flyer Xcelsior XE40 $300 \mathrm{~kW}$ ) and compare to the current CNG model (Orion $V$ ) along an actual Unitrans route. The model estimates that the selected bus can travel 146 miles on a single charge, with a fuel economy of $1.75 \mathrm{kWh}$ per mile, which meets the service requirements. Results for bus replacement schedules between 5 and 49 in the 12-year analysis period indicate that between 1600 and 22,000 MT of carbon can be avoided. The net present cost analysis indicates that the potential savings from the replacement of a single CNG bus with an electric bus (with available incentives) ranges from $\$ 146,000$ - $\$ 211,000$ per bus over its lifetime, depending on infrastructure costs.
\end{abstract}

\section{Keywords}

Electric Vehicles, Electric Bus, Economic Analysis, Greenhouse Gas, Battery Capacity, ADVISOR

\footnotetext{
"Corresponding author.
} 


\section{Background and Motivation}

As government agencies and private companies set increasingly ambitious climate action goals, the need to decarbonize the public transportation sector is great. In the United States, transportation is responsible for 27 percent of total greenhouse gas (GHG) emissions [1]. In addition to emitting carbon dioxide, internal combustion vehicles present a range of health risks from exhaust, such as respiratory irritation, lung cancer and heart disease [2]. Although hybrid vehicles have shown a $20 \%-40 \%$ reduction in the "well to wheel" emissions of $\mathrm{CO}_{2}$, hybrid technology does not offer the scale of emissions reductions that many agencies require [3]. The shift to lower GHG emissions vehicles in the private sector is predicted to be slow based on market penetration. The transformation of public transportation (i.e. buses), however, can be faster with typical vehicles having a shorter service life and often only a few decision makers needed to implement the switch.

There are three technology options that could help transit agencies meet aggressive carbon neutrality emission goals: biomethane (RNG), renewably-sourced hydrogen, and battery electric vehicles (BEVs). Biomethane has shown promise as a transportation fuel. The City of Sacramento California, for example, currently fuels refuse trucks and school buses with RNG produced from the Sacramento Biodigester. However, RNG relies on costly infrastructure, couples energy production to waste streams, and faces challenges to scale, such as sourcing feedstock. On the other hand, renewably-sourced hydrogen may be an excellent fuel source of the future, but the technology is not ready for commercial-scale deployment and is extremely costly [4]. Furthermore, the vast majority of hydrogen produced in the U.S. comes from stream reforming of natural gas, which gives hydrogen a well to wheel emissions factor similar to compressed natural gas [5]. Battery electric vehicles are ready for commercial-scale deployment, as the electrical infrastructure is largely already in place to allow charging and electricity is easily sourced from renewables [2] [3]. Battery electric buses (EBs), like their automotive counterpart, have an on-board traction battery and similarly are carbon neutral in the use phase.

University and college campuses play a critical role in reducing greenhouse gas emissions. In 2005, US colleges and universities were responsible for $2 \%$ of the country's total greenhouse gas emissions [6]. Many campuses have increased their renewable energy production and have completed energy efficiency retrofits. However, decarbonizing vehicle fleets, particularly bus fleets, remains a challenge due to the higher cost of EBs over their conventional counterparts and sunk cost agencies typically have in existing fleets and infrastructure.

At the University of California, Davis (UC Davis) Unitrans operates 20 routes, logs over 1 million miles annually, carries 4.5 passengers per vehicle mile, and owns 49 buses, most of which are fueled by CNG [7]. These operations represent less than 1 percent of the roughly 272,000 $\mathrm{MTCO}_{2} \mathrm{e}$ annual emissions campus-wide in 2013 [8]. Rather than purchasing more CNG buses, Unitrans is considering adding EBs to the fleet to save money and reduce greenhouse gas (GHG) emissions. This study investigates the economic viability and potential GHG reduction of this move and is comprised of four sections: 1) a review of currently available EB technology suitable for the Unitrans fleet, 2) an ADVISOR energy model to determine energy use, 3) an economic analysis and 4) greenhouse gas accounting.

\section{Battery Electric Bus and Charging Options}

Four leading plug-in electric bus manufacturers, BYD, Proterra, New Flyer, and Complete Coach Works, are assessed to determine if each technology meets the criteria of Unitrans. Data regarding the lifetime, range, battery capacity, bus weight, warranty, efficiency, and price of each EB were collected from manufacturer's published data as well as via phone and email correspondence with company representatives (Table 1). The data are intended to act as a general guide, as bus prices are subject to bid and model specifications. Also, many EBs have modular battery packs that are customizable to route demands that impact cost and range.

The average fuel economy of the four bus models is $1.8 \mathrm{kWh}$ per mile. Since New Flyer Xcelsior ${ }^{\circledR}$ XE40 with a $300 \mathrm{~kW}$ battery (XE40) fuel economy is closest to this value, it is considered to be a "typical" EB for this study. Thus, specifications for New Flyer are used as model inputs into ADVISOR vehicle simulation to determine energy use $(\mathrm{kWh} / \mathrm{mile})$ as well as if the range between charging meets the service requirements. 
Table 1. Plug-in electric vehicle specifications.

\begin{tabular}{cccccc}
\hline Bus Company & Price (\$) & Range (miles) & $\begin{array}{c}\text { Optional Warranty } \\
\text { (years) }\end{array}$ & $\begin{array}{c}\text { Fuel Economy } \\
\text { (kWh/mile) }\end{array}$ & $\begin{array}{c}\text { Gross Weight } \\
\text { (lbs.) }\end{array}$ \\
\hline BYD & 800,000 & $155+$ & 12 & 1.92 & 40,786 \\
Proterra & 750,000 & $96-215$ & 12 & 1.7 & 39,050 \\
New Flyer & 850,000 & $38-130$ & 12 & 1.84 & $42,540-44,312$ \\
Complete Coach Works & 650,000 & $85-115$ & 12 & $1.7-2.0$ & 37,740 \\
Average & 762,500 & $94-154$ & 12 & 1.8 & 40,251 \\
\hline
\end{tabular}

Inductive charging, the most expensive type, can be placed in-route to extend an EB's range, but the energy transfer inefficiency of the inductive charging can generate a lot of waste heat and energy. Additionally, inductive charging installation is an intensive process that involves road construction and likely includes additional political and bureaucratic obstacles. Direct contact charging is a simpler technology with higher energy transfer efficiency from the charger to the bus compared to inductive charging, but it is generally more costly than traditional plug-based charging.

For this study, only plug-based charging options are explored, andthe cost to install this type of infrastructure varies widely. Depending on where the transit agency wishes to install the station and what electrical infrastructure exists (if any), the cost for a single charging station can range from $\$ 1000$ to over $\$ 600,000$ [9]. Many EBs require a $480 \mathrm{~V}$, 3-phase power, 5-wire, 100 AMP service per charger. Some bus companies provide a charging interface with the purchase of a bus (additional interfaces cost roughly \$8000), while other companies require the agency to purchase the charging interface separately [10]. If the $480 \mathrm{~V}$ power is available, a typical installation fee is about $\$ 2000$ [10]. However, if a transit agency has maximized their power use and needs to bring additional high power lines to their depot, the cost for expansion lines can be exorbitant.

\section{Methodology}

The study utilizes the technology review in Section 2 to determine the specifications of a typical electric bus based on price, range, energy use, and weight. This typical bus profile is modeled in Advisor vehicle simulation software to determine the fuel economy (kWh per mile) over the specified drive cycle. Cost and energy use data are used to determine the net present cost (NPC) as well as the $\mathrm{CO}_{2}$ impacts for the current CNG fleet well as the electric bus scenarios. Figure 1 depicts the inputs and outputs for this study.

In the present study, three bus replacement schedules are compared $(5,13$, and 49 buses) against the current full-CNG fleet. The GHG accounting is evaluated with 3 different sources of electricity, the Western Area Power Administration (WAPA) grid mix, the current UC Davis grid blend, and a 100\% Attributed Solar grid. For the NPC analysis each scenario is evaluated at 2 levels of incentives (with and without grant funding) as well as 2 levels of infrastructure cost (low and medium).

\subsection{ADVISOR 2002 Model of Electric Buses on Unitrans Route}

ADVISOR 2002 (ADVISOR) simulation software models vehicle emissions and driving capabilities over specified routes and can be applied to buses. This model calculates the total distance travelled, the gasoline equivalent fuel economy (mpg), and the energy use breakdown of mechanical systems in the vehicle [11]. As noted, the XE40 is modeled to determine whether a typical EB is capable of completing the average Unitrans route distance on one charge.

The following assumptions are used as inputs to ADVISOR:

- Lithium ion, $300 \mathrm{~kW}$ capacity battery.

- $80 \%$ battery round trip efficiency.

- Gross weight is $15,454 \mathrm{~kg}$.

- Transmission: 85\% efficiency.

- "Heavy" wheel/axle and accessory.

- Max power is $160 \mathrm{~kW}$ MC_AC75 motor option, 92\% efficiency.

- Battery is discharged to manufactures recommended minimum capacity (20\%). 


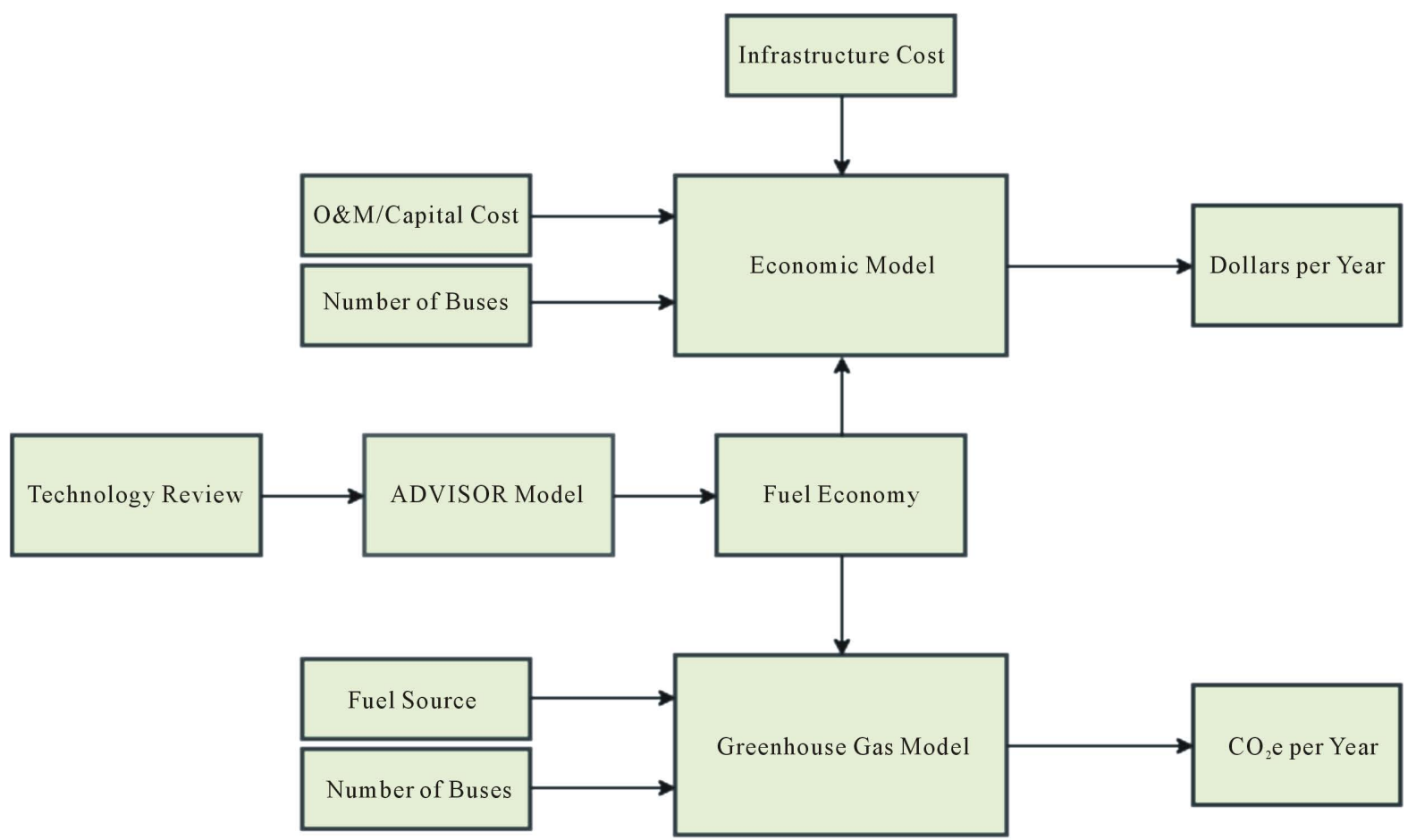

Figure 1. Model inputs and outputs.

Unitrans collects real-time data on the speed of every bus in the field. For ADVISOR, data from Unitrans "Column 50" bus route was used. Buses assigned to this route run 23 laps over the course of one day, which results in a total of 140 miles travelled. Column 50 was chosen because it represents the average distance travelled per day for all the Unitrans routes (140 miles). In ADVISOR, the XE40 was simulated on Unitrans Column 50 to determine if the bus could complete the day's route without stopping to recharge. The fuel economy is a required input for both the economic and GHG models. The fuel economy results from ADVISOR are compared to that of the manufacturer specifications for the XE40 to ensure that the published numbers correspond to the actual route used in this study.

A simulated drive cycle was created in ADVISOR (Figure 2). As shown, the bus's speed never exceeds 16 mph with 9 stops along the route. The break in the bus's motion halfway through the route represents the mandatory wait time at the bus depot before being deployed on the second half of the route.

ADVISOR predicts that the XE40 uses $1.75 \mathrm{kWh}$ per mile, while the ALTOONA test rating for the XE40 is $1.84 \mathrm{kWh}$ per mile [12]. ADVISOR results are in close agreement with the ALTOONA rating, which manufacturers use for their published figures. The slight difference in energy use could be attributed to the difference in the ALTOONA drive cycle and the Davis Column 50 route. Given the closeness in the ADVISOR and ALTOONA results, the manufacturer's efficiency ratings are used for the present study. Thus, the fuel economy input value for the GHG analysis is the average fuel economy of the four electric buses: $1.8 \mathrm{kWh}$ per mile. Along with energy efficiency, ADVISOR predicts the total energy use over column 50 to be $247 \mathrm{kWh}$, or 146 miles, corresponding to $80 \%$ of the total battery capacity.

\subsection{Assumptions of the GHG Analysis}

GHGs attributed to CNG buses are a result of in-use combustion and those for electric buses are emitted during the production of the electricity used for charging. For the GHG comparison in this study both EB and CNG buses are assumed to $\log$ 20,000 miles per year, based on 2014 Unitrans data [7]. The CNG buses are assumed to have a fuel economy of 0.4 therms per mile and emit $5.0 \times 10^{-3}$ metric tons $\mathrm{CO}_{2} \mathrm{e}$ per therm where $\mathrm{CO}_{2}, \mathrm{CH}_{4}$, and $\mathrm{N}_{2} \mathrm{O}$ emissions are converted into $\mathrm{CO}_{2} \mathrm{e}$ using USEPA conversion factors [1] [7]. The typical EB (XE40) is assumed to have a fuel economy of $1.8 \mathrm{kWh}$ per mile (see Section 2). The emissions factors for both the XE40 and CNG bus for each energy source considered in this study are summarized in Table 2. 


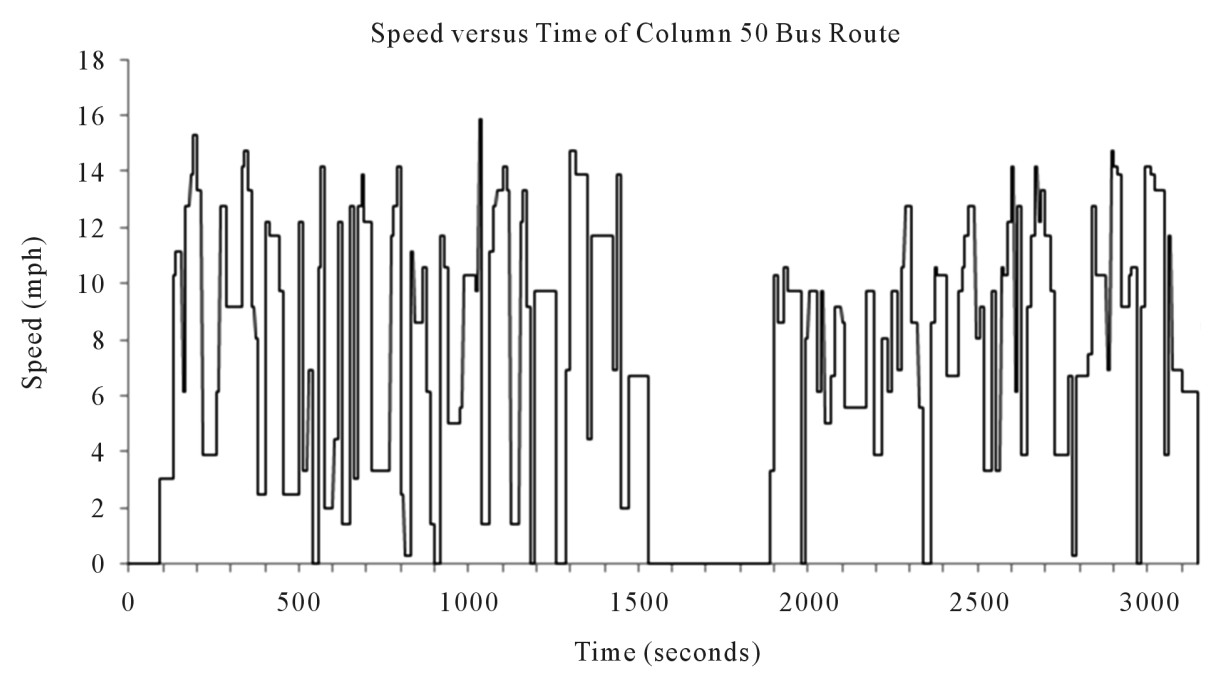

Figure 2. Graphical representation of the column 50 Unitrans bus route.

Table 2. Energy sources for battery electric and CNG buses (beginning in 2017).

\begin{tabular}{cccc}
\hline & Percent Renewable & $\begin{array}{c}\text { Percent } \\
\text { Non-Renewables }\end{array}$ & $\begin{array}{c}\text { Metric Tons } \\
\mathrm{CO}_{2} \text { e per kWh }\end{array}$ \\
\hline WAPA Grid & 30 & 70 & $3.00 \mathrm{E}-04$ \\
UC Davis Grid & 53 & 47 & $1.40 \mathrm{E}-04$ \\
$100 \%$ Solar & 100 & 0 & 0 \\
CNG Bus & -- & -- & $5.00 \mathrm{E}-03$ \\
\hline
\end{tabular}

\section{Calculations for the GHG Analysis}

The GHG analysis considers 9 scenarios. Incorporating Unitrans' bus replacement schedule, the model evaluates emissions from replacing 5, 13, and 49 buses (full fleet replacement). Under each replacement schedule, 3 different electricity sources were considered: the WAPA power grid, the current UC Davis Blend and a $100 \%$ solar powered grid. While an exclusively WAPA powered bus is not an option since the current campus blend already has significant input from on-site solar, this option demonstrates that Unitrans has additional incentive to implement EBs due to the large portion of renewable energy in their electricity portfolio.

\subsection{Calculation of Net Present Cost (NPC)}

This study compares the NPC associated with the replacement of a single CNG bus with an EB for six cases. NPC for each case are calculated by summing discounted capital, operations and maintenance, fuel, and infrastructure costs, and incentives over a 12-year analysis period. The assumptions regarding fuel economy and miles travelled per year from the GHG model are used in the economic model. This study assumed a discount rate (r) of 6 percent for all economic calculations. $C_{t}$ represents the sum of capital, fuel, and maintenance costs over a given year. The equation below was used to calculate the NPC for all scenarios.

$$
\mathrm{NPC}=\sum_{t=1}^{T} \frac{C_{t}}{(1+r)^{t-1}}
$$

Three cases are considered both with and without grant funding (incentives): 1) EB with low cost charging infrastructure; 2) EB with medium cost charging infrastructure; and 3) CNG (business-as-usual). Based on data from the California Air Resources Board, it is assumed that Unitrans could receive $\$ 431,000$ for a new CNG bus and $\$ 656,000$ for an electric bus [13]. Additional funding is available for the purchase of electric buses in California, such as $\$ 110,000$ grants awarded by Hybrid and Zero Emission Truck and Bus Voucher Incentive Project (HVIP), but for conservative estimates, we omitted these additional grants from the economic analysis. 
A new CNG bus is assumed to cost $\$ 600,000$ in 2016 [7]. The price per EB is estimated as the average cost of the four EBs considered: $\$ 762,500$. A conservative maintenance cost of $\$ 0.40$ per mile is assumed for EBs (other sources indicate EB maintenance costs may be significantly lower) [14]. Alternatively, a maintenance cost of $\$ 0.60$ per mile for the CNG buses is assumed [7]. Forecasted electricity and natural gas prices are included and shown in Figure 3 [15].

\section{Infrastructure Costs}

For the additional infrastructure costs associated with replacement of CNG buses with EBs low and medium cost scenarios are considered ${ }^{1}$ in the present study. The low scenario consists of limited electrical infrastructure costs $(\$ 30,000$ per charging site) with no additional cost for the charging interface. In contrast, the medium scenario consists of an electrical infrastructure cost $(\$ 50,000)$ with an additional cost $(\$ 45,000)$ for each charging interface. These two infrastructure cost structures are determined by estimates from a utilities specialist on the UC Davis campus and the various charging packages offered by EB companies [16]. The cost structure assumes two buses per interface, which follows a process of charging one bus completely and then transferring the plug to another bus over the course of a night. Thus, the infrastructure costs associated with the replacement of one bus are assumed to be $\$ 30,000$ and $\$ 95,000$ for the low and medium scenarios respectively. For the present study infrastructure costs associated with the CNG bus are assumed to be zero since all the necessary components already exist on the UC Davis campus

The proposed site can only support charging four buses at a time; so if Unitrans were to add more than four EBs to the fleet, they would need an additional charging site and incur further infrastructure-related costs. Under the following assumptions some economy of scale may be realized in reduced infrastructure costs, as shown in Table 3, but in order to accurately predict the electrical carrying capacity of the proposed charge site, a permanent meter at the site would be needed to show historical peak load values.

\section{Results}

\subsection{GHG Emission Results}

A new CNG bus, under the above assumptions, would produce approximately 38 metric tons of $\mathrm{CO}_{2} \mathrm{e}$ per year. If all 49 Unitrans buses remain CNG buses, the emissions would be approximately 1856 metric tons of $\mathrm{CO}_{2} \mathrm{e}$ per year. This is shown as the red bar in the bottom of Figure 4.

If charged on the WAPA grid, each electric bus will produce approximately 11 metric tons of $\mathrm{CO}_{2} \mathrm{e}$ a year, a savings of 27 metric tons of $\mathrm{CO}_{2} \mathrm{e}$ emissions when compared to a single CNG bus. If the 5 buses to be replaced in 2016 are electric buses, the emissions will be approximately 1720 metric tons of $\mathrm{CO}_{2}$ e per year. With 13 electric buses in the 2019 scenario emissions will be approximately 1504 metric tons of $\mathrm{CO}_{2} \mathrm{e}$ in a year. Finally, if

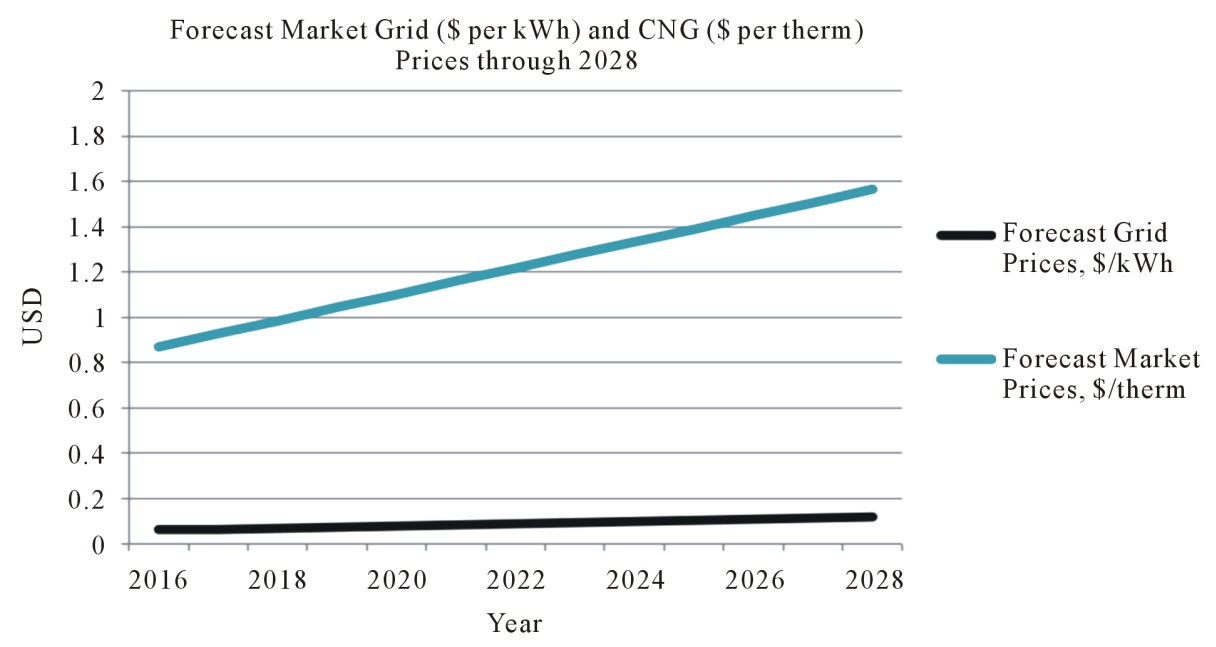

Figure 3. Forecast market grid and CNG prices through 2028 [15].

\footnotetext{
${ }^{1} \mathrm{~A}$ high cost scenario with infrastructure of $\$ 2$ million was excluded from the analysis.
} 
all 49 buses are replaced with new electric buses and charged on WAPA power, fleet emissions will be approximately 529 metric tons of $\mathrm{CO}_{2} \mathrm{e}$ in a year.

For each scenario, the GHG savings over the 12-year life span is calculated and are shown in Table 4 below. The savings are measured against the business as usual scenario where all buses are to be replaced with new CNG buses.

The existing UC Davis Blend is the most likely charging source for Unitrans. In this scenario, a single electric bus charged will emit approximately 5 metric tons of $\mathrm{CO}_{2} \mathrm{e}$ in a year. This scenario provides a reduction of 33 metric tons of $\mathrm{CO}_{2} \mathrm{e}$ in a year when compared to the currently used CNG buses and 6 metric tons $\mathrm{CO}_{2} \mathrm{e}$ when

Table 3. Infrastructure cost estimates.

\begin{tabular}{|c|c|c|c|c|c|c|}
\hline \multirow{2}{*}{ Number of buses } & \multirow{2}{*}{$\begin{array}{l}\text { Required charging } \\
\text { interfaces }\end{array}$} & \multirow{2}{*}{$\begin{array}{c}\text { Required } \\
\text { charging sites }\end{array}$} & \multicolumn{2}{|c|}{ Low Scenario } & \multicolumn{2}{|c|}{ Medium Scenario } \\
\hline & & & $\begin{array}{l}\text { Infrastructure } \\
\text { cost }(\$)\end{array}$ & $\begin{array}{l}\text { Infrastructure } \\
\text { cost (\$/bus) }\end{array}$ & $\begin{array}{l}\text { Infrastructure } \\
\text { cost }(\$)\end{array}$ & $\begin{array}{c}\text { Infrastructure } \\
\text { cost (\$/bus) }\end{array}$ \\
\hline 1 & 1 & 1 & $\$ 30,000$ & $\$ 30,000$ & $\$ 95,000$ & $\$ 95,000$ \\
\hline 2 & 1 & 1 & $\$ 30,000$ & $\$ 15,000$ & $\$ 95,000$ & $\$ 47,500$ \\
\hline 3 & 2 & 1 & $\$ 30,000$ & $\$ 10,000$ & $\$ 140,000$ & $\$ 46,667$ \\
\hline 4 & 2 & 1 & $\$ 30,000$ & $\$ 7500$ & $\$ 140,000$ & $\$ 35,000$ \\
\hline 5 & 3 & 2 & $\$ 60,000$ & $\$ 12,000$ & $\$ 235,000$ & $\$ 47,000$ \\
\hline 13 & 7 & 4 & $\$ 120,000$ & $\$ 9231$ & $\$ 515,000$ & $\$ 39,615$ \\
\hline 49 & 25 & 13 & $\$ 390,000$ & $\$ 7959$ & $\$ 1,775,000$ & $\$ 46,020$ \\
\hline
\end{tabular}

Table 4. Projected $\mathrm{CO}_{2}$ savings over the 12-year analysis period.

\begin{tabular}{cccc}
\hline & $\begin{array}{c}\text { MTCO } \\
\text { with } 5 \text { of } 49 \text { EBs }\end{array}$ & $\begin{array}{c}\text { MTCO }_{2} \text { e Savings } \\
\text { with } 13 \text { of } 49 \text { EBs }\end{array}$ & $\begin{array}{c}\text { MTCO }_{2} \text { Savings } \\
\text { with 49 EBs }\end{array}$ \\
\hline WAPA Grid & 1624 & 4223 & 15,918 \\
UC Davis Blend & 1965 & 5110 & 19,259 \\
$100 \%$ Attributed Solar & 2272 & 5908 & 22,268 \\
\hline
\end{tabular}

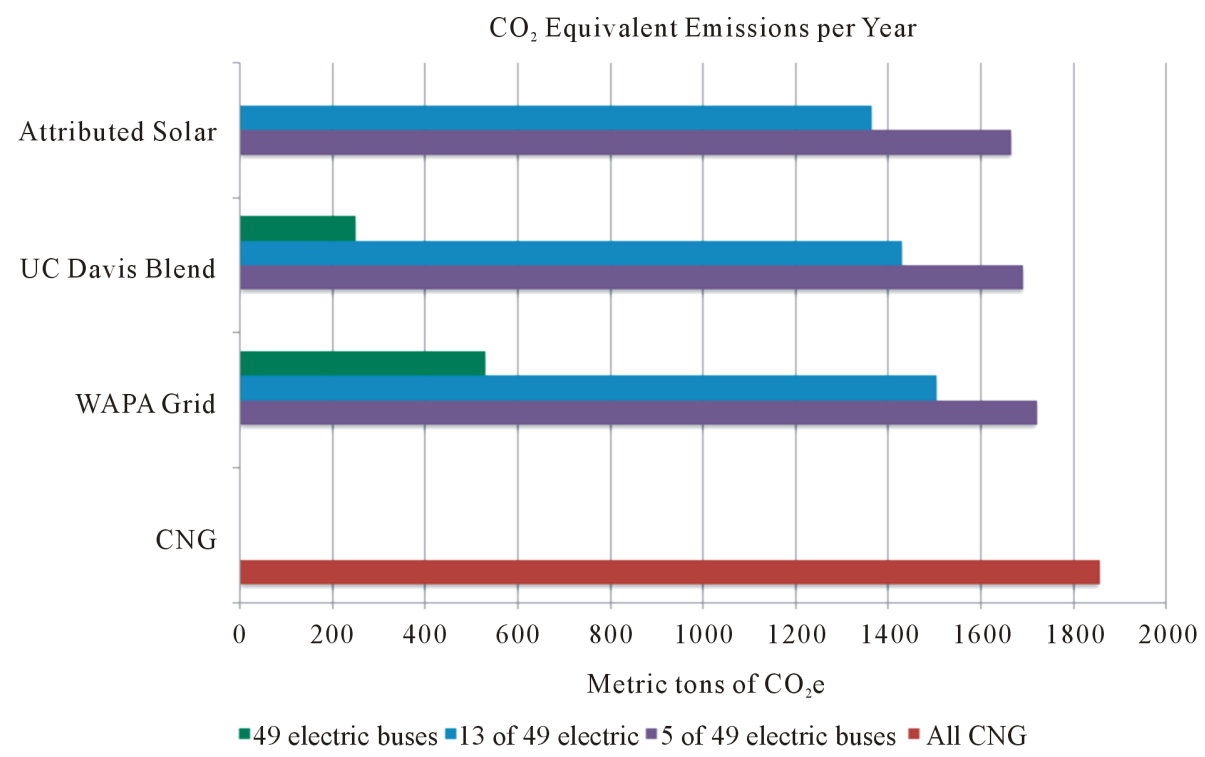

Figure 4. $\mathrm{CO}_{2}$ equivalent emissions per year for 4 scenarios. 
compared to an electric bus charged on the conventional WAPA grid. With 5 EBs, the fleet will emit approximately 1692 metric tons of $\mathrm{CO}_{2} \mathrm{e}$ per year. With $13 \mathrm{EBs}$, the fleet will emit approximately 1430 metric tons of $\mathrm{CO}_{2} \mathrm{e}$ in a year. Finally, with all $49 \mathrm{EBs}$ charged on the UC Davis grid, the fleet would emit 251 metric tons of $\mathrm{CO}_{2} \mathrm{e}$ in a year. Charging on the UC Davis grid, due to its higher proportion of renewables, provides significant carbon emission benefits for the Unitrans fleet.

The optimum charging scenario for GHG emissions reductions will occur if EBs in the Unitrans fleet charge on electricity allocated from the large off-site solar project. In this scenario, all EBs would have zero $\mathrm{CO}_{2} \mathrm{e}$ emissions for the duration of their use. All fleet emissions would come from remaining CNG buses.

\subsection{Results of the Economic Analysis}

The NPC results for the six cases for replacement of a single bus are presented in Table 5 below. As shown, grant funding to offset the purchase price has a large impact on the net present cost of the project for both CNG and electric options. The CNG scenario provides the lowest cost choice if grant funding is not pursued; however, if all potential grants are included, the EBs have a lover net present cost over the 12-year analysis period in Figure 5.

\section{Discussion}

The methodology presented here provides a basis to evaluate if a switch from CNG to electric buses is viable from both an economic and GHG prospective.

Although the Advisor model as well as manufactures figures suggest that many of the current EB offerings would meet the service requirements it should be noted that in many instances the range between charges can be reduced by adverse weather conditions, the use of heat or air conditioning and other accessories, as well as lowered battery capacity with time (number of cycles). In order to compensate for these variables, initial bus battery pack may need to be upsized. Actual data and a better understanding of real world conditions will allow for a better range estimation and the selection of an appropriate bus that can meet the requirements under all conditions.

Table 5. NPC with and without grant funding including infrastructure costs.

\begin{tabular}{ccc}
\hline & NPC (Without Grant Funding) & NPC (With Grant Funding) \\
\hline EB: Low Infrastructure & $\$ 981,737$ & $\$ 325,737$ \\
EB: Medium Infrastructure & $\$ 1,046,737$ & $\$ 390,737$ \\
CNG Bus & $\$ 968,120$ & $\$ 555,120$ \\
\hline
\end{tabular}

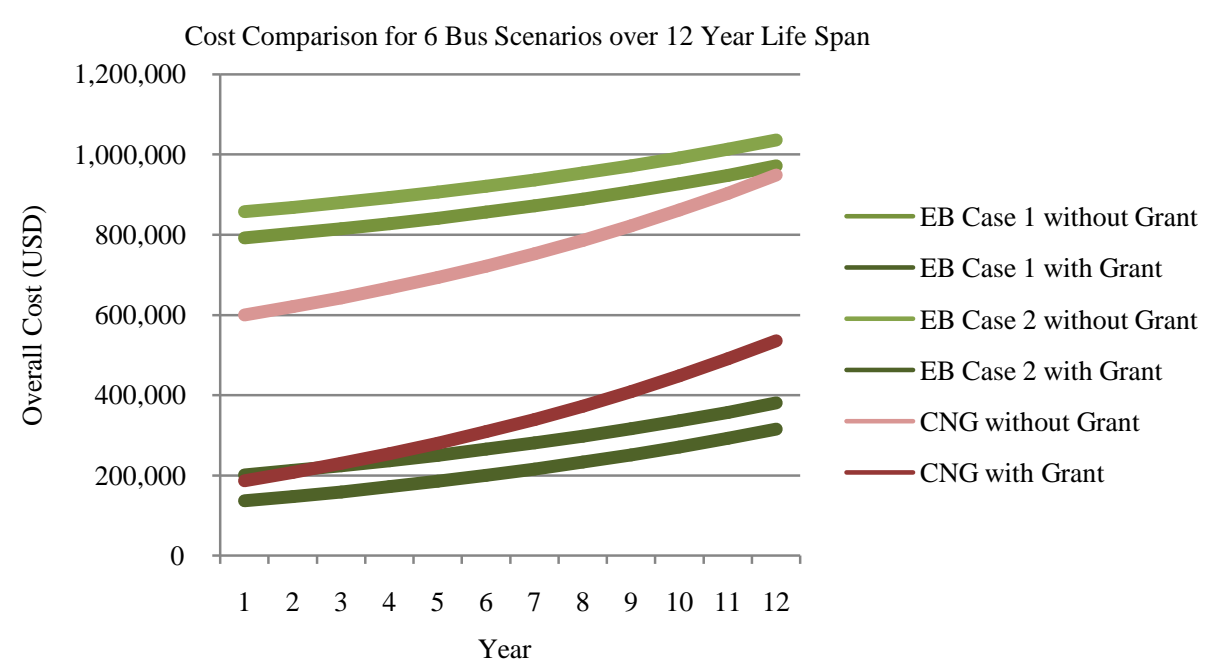

Figure 5. Net present cost comparison for a single bus over the 12-year analysis period. 
The UC Davis Grid blend has a high renewable fraction thus GHG results are specific to the campus. Thus, the WAPA grid GHG results are more applicable estimates for most other institutions. Under WAPA conditions, switching to EBs will provide a savings of approximately 325 metric tons of $\mathrm{CO}_{2} \mathrm{e}$ per bus replaced. Depending on the number of CNG buses replaced, an institution's emissions related to transportation could be greatly reduced.

Additionally, because EB technologies are in their nascent stage, it is difficult to predict maintenance costs for the vehicles. The economic analysis includes a conservative number for the EB maintenance cost ( $\$ 0.40 /$ mile), however EB maintenance cost could be as low as $\$ 0.15$ per mile, which would significantly shift the results of the analysis in favor of EBs [17].

If significant incentives for EBs are available to offset the purchase price, a good economic case can be made for the transition as shown in this case study. In California, where UC Davis is located, there are two primary funding streams for EBs. The California Hybrid and Zero-Emission Truck and Bus Voucher Incentive Project (HVIP), designed to speed market adoption of low carbon vehicles, offers a 4 year preliminary voucher up to $\$ 117,000$ for qualified EBs [18]. Additionally, the California Air Resources Board has allocated \$45 million, under the Zero-Emission Truck and Bus Pilot Commercial Deployment Project, to drive down electric bus costs and help meet emission reduction targets. However, in the U.S., incentives for EBs vary considerably by state. Since the grant funding seriously impacts the economic viability of bus replacement, institutions pursuing an all-electric fleet should investigate available incentives.

\section{Conclusions and Recommendations}

Greenhouse gas emissions and cost analyses for EB fleet conversion is highly dependent on the carbon intensity of the available electricity grid, the fleet driving characteristics (e.g. route distances), maintenance costs, preexisting infrastructure and the purchase price. Also, transit agencies looking to add EBs to their fleet must also consider the vender reputation, powertrain, chassis and component longevity, part availability, options for extending range, and data collection hardware-all key factors of decision-making for a transit agency that are not included in the scope of this report. Further investigation is needed to better quantify infrastructure costs and battery capacity depletion over time. Site-specific measurements and analysis must be made to determine the actual cost of implementing charging infrastructure. Finally, additional research might consider the impacts of including in-route charging for partial or entire fleet use.

Although the results of this study are specific to the Unitrans case on the campus of the University of California Davis, some insights can be applied to other transit agencies:

- The current offering of battery electric bus products can meet the service requirements for a campus transit agency. Three of the four electric buses reviewed in this report have the range and charging capacity to meet Unitrans' needs without the use of in-route charging.

- ADVISIOR 2002 can be used to predict battery electric buses range and fuel efficiency over specific driving conditions. Specifically, the performance for the XE40 was successfully simulated on the UC Davis Column 50 route.

- The potential for GHG reductions associated with battery electric buses are highly dependent on the electricity source. At UC Davis, if the buses use power attributed to solar electricity, the bus and its fuel would have zero greenhouse gas emissions associated with the use phase. Even without attributed solar, UC Davis GHG emissions are reduced by 22.7 metric tons of $\mathrm{CO}_{2} \mathrm{e}$ when one electric bus is charged on the current UC Davis grid blend.

- Emissions reduction potential associated with battery electric buses are significant but may be small relative to total GHG emissions. As seen in the UC Davis case under current conditions, the adoption of an entire EB fleet result in a 7 -fold reduction in GHGs for Unitrans, which only accounts for less than $1 \%$ of the total campus scope 1 and 2 emissions.

- Charging infrastructure costs associated with electric buses depend on fleet size and existing electrical infrastructure and thus must be determined on a case-by-case basis. In the UC Davis case estimates for infrastructure costs vary widely and may have an economy of scale with the cost per electric buses decreasing as the number of EBs in the fleet increases. Estimates for the infrastructure cost from the above analysis range from $\$ 95,000$ (one bus under the medium scenario) to $\$ 8000$ (49 buses under the low scenario) per bus.

- Electric buses may have lower lifetime costs compared to their CNG counterparts, with fuel and mainten- 
ance cost savings as well as available incentives playing a major role. Results from the UC Davis study indicate a potential net present cost savings of $\$ 211,000$ per bus over its lifetime with grant funding.

\section{Highlights}

- Inventory of currently available electric bus models and associated government incentives.

- Net present cost is calculated for a CNG and an electric bus over a 12-year life.

- ADVISOR modeling is used to demonstrate electric bus energy consumption over UC Davis route.

- Greenhouse gas analysis to determine carbon savings from switching to electric buses under different power generation mixes.

- Electric bus charging infrastructure concerns and costs.

\section{Acknowledgements}

The authors would like to acknowledge Katrina Sutton, Jessica Siegel, Camille Kirk, Brian Leung, Melody Lin, Damon Williams and Gil Tal for their help in the preparation of this paper as well as Anthony Palmere and Andrew Wyly of Unitrans for their time and assistance in obtaining the necessary data for this study. We would also like to acknowledge the National Science Foundation's PIRE (Partnership for International Research and Education) program for their support of this research.

\section{References}

[1] USEPA (2015) Sources of Greenhouse Gas Emissions. http://www3.epa.gov/climatechange/ghgemissions/sources/transportation.html

[2] Noel, L. (2014) A Cost Benefit Analysis of a V2G-Capable Electric School Bus Compared to a Traditional Diesel School Bus. Applied Energy, 126, 246-265. http://dx.doi.org/10.1016/j.apenergy.2014.04.009

[3] Miles, J. (2014) Developing a Viable Electric Bus Service: The Milton Keynes Demonstration Project. Research in Transportation Economics, 48, 357-363. http://dx.doi.org/10.1016/j.retrec.2014.09.063

[4] Abbasi, T. (2011) "Renewable” Hydrogen: Prospects and Challenges. Renewable and Sustainable Energy Reviews, 15, 3034-3040. http://dx.doi.org/10.1016/j.rser.2011.02.026

[5] Ruth, M. (2009) Hydrogen Pathways: Cost, Well-to-Wheels Energy Use, and Emissions for the Current Technology Status of Seven Hydrogen Production, Delivery, and Distribution Scenarios. NREL Technical Report, NREL.

[6] Sinha, P., et al. (2010) Greenhouse Gas Emissions from U.S. Institutions of Higher Education. Journal of the Air \& Waste Management Association, 60, 568-573. http://dx.doi.org/10.3155/1047-3289.60.5.568

[7] Palmere, A. (2015) Interview with Anthony Palmere. In: Mickle, C., Ed.

[8] Kirk, C. (2015) Details of UC Davis Emissions. In: Mickle, C., Ed.

[9] Thomas, W. (2015) Discussion Regarding Electric Buses at Standford University. In: Mickle, C., Ed.

[10] Kahn, Z. (2015) UC Davis: Bus Charging and Specifications. In: Mickle, C., Ed.

[11] Gao, W.Z. (2007) Modeling and Simulation of Electric and Hybrid Vehicles. IEEE.

[12] Altoona (2015) Federal Transit Bus Test-New Flyer XE40. US DOT, 1-128.

[13] ACT (2015) Advanced Clean Transit. In: Board, A.R., Ed.

[14] Wyley, A. (2015) Correspondence with Andrew Wyley, Unitrans Maintenance Manager. In: Mickle, C., Ed.

[15] Cook, J. (2014) The Future of Electricity Prices in California: Understanding Market Drivers and Forecasting Prices to 2040. UC Davis Energy Efficiency Center, 1-37.

[16] Williams, D. (2015) Email Correspondence between Damon Williams and Colin Mickle Regarding Electric Bus Charging Cost. In: Mickle, C., Ed.

[17] Thomas, W. (2015) Electric Buses at Standford. California Higher Education Sustainability Conference.

[18] CAHVIP (2015) Electriv Vehicle Specification Sheets. California Hybrid and Zero-Emission Truck and Bus Voucher Incentive Project. 